\title{
Commentary on the history and quantitative nature of filter paper used in blood collection devices
}

It is with great interest that we read Peter T Kissinger's commentary about the use of dried blood spots (DBS) for pharmacokinetic assays and therapeutic drug monitoring published in the October issue of Bioanalysis [1]. We appreciate Kissinger's efforts to stimulate debate regarding the utility of DBS in these two types of applications. However, we would like to dispel some misconceptions about DBS and their filter paper matrix that readers, unaware of their many complexities, may derive from his commentary. Our intent is to provide information supporting the use of DBS in many applications, based on 50 years of DBS use by newborn screening (NBS) laboratories in the USA.

\section{DBS: $\mathbf{5 0}$ years of knowledge about the filter paper matrix}

Neonatal screening activities were initiated when Guthrie and Susi first introduced a simple method for the analysis of phenylalanine in DBS to detect phenylketonuria in population screening [2]. Today NBS is the largest population-based genetic screening effort in the USA [3], and relies on the use and ease of transport of filter paper blood collection devices. Kissinger's assertion that newborn screening assays are mostly MS-based and generally qualitative, does not reflect current practice, which relies on the use of DBS punches as a quantitative measure with a well-characterized variability. While most biomarkers assayed in NBS can be identified through MS-based assays, the majority of the assays involve colorimetric and fluorometric determinations of various biomarkers indicative of several inborn errors of metabolism [4]. It is the quantitative nature of this matrix that has allowed the establishment of cut-off values by NBS programs to differentiate asymptomatic newborns that may have a disease from those who may not. The small variability of the filter paper matrix has prevented the standardization of DBS use in clinical settings. This underscores the importance of understanding the nature of the filter paper matrix, including its limitations, via a review of the existing literature generated by decades of NBS activities worldwide.
The filter paper matrix is designed to absorb a specific volume of blood and therefore, although less precise than a pipette, has a volume associated with a given area of the card. In the USA, only papers from sources approved by the FDA are acceptable for blood collection for clinical tests. Critical to the proper use of this matrix is an ongoing assessment of new lots as they are manufactured. Since the punch selected from a DBS is used as a volumetric sample for quantitative analysis, a high degree of uniformity is essential to intra-production lot and inter-lot performance for calibrators, quality control materials and unknown samples. The Centers for Disease Control and Prevention's Newborn Screening Quality Assurance Program (NSQAP) has evaluated filter paper lots of Grade $903^{\circledR}$ paper for over 30 years [101]. The published acceptable serum-absorption volume per $1 / 8$ inch disk punched from a $100 \mu \mathrm{l}$ spot of intactcell blood of $55 \%$ hematocrit is $1.54 \pm 0.17 \mu \mathrm{l}$ [5]. Furthermore, many other peer-reviewed reports have described the effects of hematocrit on quantitative measures from DBS [6], as well as differences between filter paper manufacturers obtained from careful study of different types of analytes in DBS [7]. The latter study's data indicated that the difference in analytical results between manufacturers could be at least 4-5\% for comparability or, at a minimum, equal to the lot-to-lot variance of a single manufacturer's filter paper products. To suggest that DBS cannot be used as a volumetric measure is not consistent with published knowledge in the NBS field, and does a disservice to the expansion of DBS applications in nonscreening environments, such as pharmacokinetics and therapeutic drug monitoring.

While DBS collection does not require highly accurate sampling, it does have its challenges. The convenience of sample storage and shipment is not to be underestimated, but the quantitation is robust especially since most NBS laboratories define errors of $15 \%$ or less as acceptable. In terms of animal welfare - we cannot speak to that - but what seems missing from the discussion is the human component. Sampling from an infant is no easy task and does cause pain. Collecting $2-5 \mathrm{ml}$ of blood is particularly
Víctor R De Jesús*1 \& Donald H Chace ${ }^{2}$

'Biochemical Mass Spectrometry Laboratory, Centers for Disease Control \& Prevention, 4770 Buford Highway, NE, Mail Stop F-19, Atlanta, GA 3034I, USA ${ }^{2}$ Pediatrix Analytical, The Center for Research \& Education, Pediatrix Medical Group, Inc., FL, USA *Author for correspondence: E-mail: vdejesus@cdc.gov 
difficult in a preterm low birth weight (LBW) baby. The advantages of DBS outweigh the problems associated with venipuncture sampling, including the reduction of contamination with total parenteral nutrition and/or other intravenous medications. Thus, assays and techniques developed in animal studies using DBS may also apply to LBW infants. The idea that some bioactive metabolites will be affected by collecting a spot applies both to animals and humans. Considering the years that DBS have been used, stating that DBS offers no sampling advantages is somewhat shortsighted, especially since no one has suggested that DBS will replace liquid samples.

Kissinger offers an interesting assessment of $3 \mathrm{D}$ versus $2 \mathrm{D}$ absorbent matrices. The filter paper blood collection device is not $2 \mathrm{D}$, but rather 3D. Although the thickness is smaller than a packed column, laboratorians can purchase filter papers with different thicknesses designed to absorb more liquid sample. Also, is there really a difference if you were to pack the filter paper in a tube and add liquid or spot it? We think not, provided specimens are dried. Sonication or vigorous shaking is not recommended because the paper fibers are dislodged, causing potential for clogging in instrument solvent and sample lines. A microtube in an envelope would still not go through the automated sorting devices at the post office. It is also known that dry specimens tend to be inactive and not reactive. However, temperatures at or below room temperature are best for some labile biomarkers, including proteins as described below.

\section{DBS analyte stability \& its utility in therapeutic drug monitoring \& clinical trials}

The stability of many common metabolites in DBS has been presented in many peer-reviewed reports. Kissinger's assertion that classical analytes, such as glucose may disappear during the drying process demonstrates the author's lack of understanding regarding biomarker behavior in DBS, and shows the need for a thorough review of the literature. While we are not aware of any studies that examine prostaglandins in DXS ( $\mathrm{X}=$ semen in this case), we would like to refer Kissinger to our analysis of dextrose in DBS. We showed through controlled experiments with stable isotope-labeled MS analysis of dextrose that its presence in DBS is not only detectable during NBS assays, but indicates improper sample collection from very LBW infants receiving high concentrations of amino acids from total parenteral nutrition administration [8,9]. Indeed, many biochemical markers and drug metabolites have been shown to be stable in DBS [10-12], which is a key advantage of DBS over liquid blood. Kissinger also presumes analytes such as enzymes would somehow be inactivated upon drying. In fact, many proteins are easily recovered and assayed from DBS, including, but not limited to, lysosomal enzymes [13], galactose-6-phosphate uridyltransferase [14] and hemoglobins [15]. As with any new assay, biomarker stability in matrix needs to be established, including the effects of storage/shipping temperature, where there is no a priori knowledge.

We agree with Kissinger's opinion regarding the utility of DBS for early-stage drug trials. However, clinical trials using filter paper have been performed [16], thus the concept of using DBS for this particular application cannot be entirely ruled out. The author's opinions on shipping may be appropriate also for drug trials but not in clinical analysis - as shown in NBS - where the cost savings are enormous. The relevant point here is not weight, but rather that liquid samples may have to be shipped on dry ice, which may be hazardous and raises cost substantially.

\section{Conclusion}

The use of DBS in newborn screening has opened the doors to a field of study that deserves the attention it is receiving in current literature. This technology, in use for decades, is well understood and characterized. Newer variations of the filter paper matrix include different chemical coatings and additives, which need to be fully evaluated by the end users in order to establish their validity for the intended application. These evaluations should take advantage of the information available regarding Grade 903 paper (and its equivalents) so as to not 'reinvent the wheel'. We agree with Kissinger when he opines that unambiguous direct measurements off a spot in 2011 would be feasible 'when pigs fly'. However, given our knowledge of DBS, we are more optimistic than he is regarding the potential of DBS to provide significant advantages to sampling and assaying drug metabolites during clinical trials compared with conventional sample handling. We do not believe DBS will supplant liquid samples; DBS may be used as 
long as proper validations steps are taken to ensure their suitability. Ultimately, DBS are worth the trouble, as they have enabled decades of population-based testing to the benefit of humankind.

\section{Disclaimer}

The findings and conclusions in this report are those of the authors and do not necessarily represent the views of the CDC.
Financial \& competing interests disclosure The authors have no relevant affiliations or financial involvement with any organization or entity with a financial interest in or financial conflict with the subject matter or materials discussed in the manuscript. This includes employment, consultancies, honoraria, stock ownership or options, expert testimony, grants or patents received or pending, or royalties. No writing assistance was utilized in the production of this manuscript.

\section{References}

1 Kissinger PT. Thinking about dried blood spots for pharmacokinetic assays and therapeutic drug monitoring. Bioanalysis 3(20), 2263-2266 (2011).

2 Guthrie R, Susi A. A simple phenylalanine method for detecting phenylketonuria in large populations of newborn infants. Pediatrics 32, 338-343 (1963)

3 De Jesus VR, Mei JV, Bell CJ, Hannon WH. Improving and assuring newborn screening laboratory quality worldwide: 30-year experience at the Centers for Disease Control and Prevention. Semin. Perinatol. 34(2), 125-133 (2010).

4 Watson MS, Lloyd-Puryear MA, Mann MY, Rinaldo P, Howell RR. Newborn screening: toward a uniform screening panel and system. Genet. Med. 8 (5 Suppl.), 12S-252S (2006).

5 Hannon WH, Whitley RJ, Davin B et al. Blood collection on filter paper for newborn screening programs; approved standard. Fifth Edition. Clinical Laboratory Standards Institute (LA4-A5), Wayne, PA, USA (2007).

6 Mei JV, Alexander JR, Adam BW, Hannon WH. Use of filter paper for the collection and analysis of human whole blood specimens. J. Nutr. 131(5), 1631S-1636S (2001).

7 Mei JV, Zobel SD, Hall EM, De Jesus VR, Adam BW, Hannon WH. Performance properties of filter paper devices for whole blood collection. Bioanalysis 2(8), 1397-1403 (2010).

8 Chace DH, De Jesus VR, Lim TH, Hannon WH, Spitzer AR. Tandem mass spectrometric identification of dextrose markers in dried-blood spots from infants receiving total parenteral nutrition. Clin. Chim. Acta 411(21-22), 1806-1816 (2010).

9 Chace DH, De Jesus VR, Lim TH, Hannon WH, Clark RH, Spitzer AR. Detection of TPN contamination of dried blood spots used in newborn and metabolic screening and its impact on quantitative measurement of amino acids. Clin. Chim Acta 412(15-16), 1385-1390 (2011).

10 Adam BW, Hall EM, Sternberg $M$ et al. The stability of markers in dried-blood spots for recommended newborn screening disorders in the United States. Clin. Biochem. 44(17-18), 1445-1450 (2011).

11 Liu G, Ji QC, Jemal M, Tymiak AA, Arnold ME. Approach to evaluating dried blood spot sample stability during drying process and discovery of a treated card to maintain analyte stability by rapid on-card $\mathrm{pH}$ modification. Anal. Chem. 83(23), 9033-9038 (2011).

12 Bowen CL, Hemberger MD, Kehler JR, Evans CA. Utility of dried blood spot sampling and storage for increased stability of photosensitive compounds. Bioanalysis 2(11), 1823-1828 (2010).

13 De Jesus VR, Zhang XK, Keutzer J et al. Development and evaluation of quality control dried blood spot materials in newborn screening for lysosomal storage disorders. Clin. Chem. 55(1), 158-164 (2009).

14 Fujimoto A, Okano Y, Miyagi T, Isshiki G, Oura T. Quantitative Beutler test for newborn mass screening of galactosemia using a fluorometric microplate reader. Clin. Chem. 46(6 Pt 1), 806-810 (2000).

15 Garrick MD, Dembure P, Guthrie R. Sickle-cell anemia and other hemoglobinopathies. Procedures and strategy for screening employing spots of blood on filter paper as specimens. N. Engl. J. Med. 288(24), 1265-1268 (1973).

16 Clark RH, Chace DH, Spitzer AR. Effects of two different doses of amino acid supplementation on growth and blood amino acid levels in premature neonates admitted to the neonatal intensive care unit: a randomized, controlled trial. Pediatrics 120(6), 1286-1296 (2007).

\section{Website}

101 Newborn Screening Quality Assurance Program annual report. www.cdc.gov/labstandards/nsqap.html 